\title{
MAXILLOFACIAL TRAUMA MANAGEMENT IN POLYTRAUMATIZED PATIENTS - THE USE OF ADVANCED TRAUMA LIFE SUPPORT (ATLS) PRINCIPLES
}

\author{
Elitsa G. Deliverska, Lachesar P. Stefanov. \\ Department of Oral and Maxillofacial surgery, Faculty of Dental Madicine, \\ Medical University, Sofia, Bulgaria.
}

\begin{abstract}
ABSTACT:
Management of the multiply injured patient requires a co-ordinated multi-disciplinary approach in order to optimise patients' outcome. A working knowledge of the sort of problems these patients encounter is therefore vital to ensure that life-threatening injuries are recognised and treated in a timely pattern and that more minor associated injuries are not omitted. This article outlines the management of polytraumatized patients using the Advanced Trauma Life Support (ATLS) principles and highlights the areas of specific involvement of the engaged medical team. Advanced Trauma Life Support is generally regarded as the gold standard and is founded on a number of well known principles, but strict adherence to protocols may have its drawbacks when facial trauma co-exists. These can arise in the presence of either major or minor facial injuries, and oral and maxillofacial surgeons need to be aware of the potential problems.
\end{abstract}

Key words: ATLS; fractures; maxillofacial; trauma

Maxillofacial trauma is without doubt a most challenging area within the specialty of oral and maxillofacial surgery. Despite the many advances in our understanding of tissue healing, biomaterials and surgical techniques, the initial assessment and the timing and undertaking of management of facial injuries in the early stages have remained a difficult area of patients' care. Appropriate and timely management of facial injuries becomes even more challenging following high velocity trauma, when significant injuries elsewhere may, or may not, take priority.(5)

This reinforces the concept of the mechanism of injury, which supports screening for the following injuries.

1. High-velocity impacts (e.g. motor vehicle collision at a pedestrian, ejection from vehicle, airbag deployment, fatalities or severe injuries to other vehicle occupants).

2. Falls from a height (typically greater than the height of the individual or $>6$ feet).
3. In patients with pelvic fractures or lower limb long-bone fractures. (6)

Familiarity with an algorithm for the assessment and initial treatment of traumatized patients provides the treating physician with the confidence to competently manage polytrauma, and ensures optimal outcome for the patient. Advanced trauma life support (ATLS) is a system of simultaneous assessment and treatment of multiple trauma patients.1 It is based on identification and safe initial management of all injuries.

In particular, it prioritizes diagnosis and effective management of life-threatening injuries. Having originally been conceived in Nebraska in the 1970s, its principles are now taught in courses all over the world and form the cornerstone of management of the patient with multiple trauma. Since its inception in 1978, the Advanced Trauma Life Support (ATLS) $(1,2,3)$ system of care has generally become accepted as the gold standard in the initial management of the multiply injured patient and is now taught in over 40 countries worldwide. This approach is based on the three well established principles of:

1. ABCDEs of assessment (Airway maintenance with cervical spine protection, Breathing with ventilation, Circulation with haemorrhage control, Disability; neurological status, and Exposure/ Environment);

2. "primum non nocere" (first, do no harm); and

3. treatment of life-threatening injuries within the 'golden hour'. The aim of the following information is to outline the acute assessment and treatment of polytraumatized patients, with an added emphasis on the management of MF injuries(6).

\section{Initial evaluation and treatment}

In the setting of a major trauma unit, the management of seriously injured patients involves the coordinated approach of a multidisciplinary team with simultaneous assessment and treatment of a variety of life-threatening conditions.

The care of the patient after hospital admission is 
divided into the following phases(7):

1. Primary survey.

2. Adjuncts to primary survey and resuscitation.

3. Secondary survey (head-to-toe evaluation and history).

4. Adjuncts to the secondary survey.

5. Continued post-resuscitation monitoring and reevaluation.

6. Definitive care.

A history of the accident and the circumstances may provide important clues to the likely pattern of injury, but in many multiple trauma patients a standard medical history cannot be obtained, due to the severity of the injuries and a reduced level of consciousness. However, it is usually possible to obtain some important items of information from relatives or paramedics if the patient is unable to provide information. The following key points of information should be obtained if possible (the ATLS mnemonic is 'AMPLE').

- Allergies.

- Medications.

- Past illnesses/pregnancy.

- Last meal.

- Events/environment of injury.

The ATLS system divides the initial assessment into a primary and secondary survey. The primary survey aims to identify immediate life-threatening injuries. The secondary survey aims to identify all other injuries that will require treatment but are not immediately life-threatening. The mnemonic for the primary survey is given by the letters ABCDE.

- Airway maintenance with cervical spine protection.

- Breathing and ventilation.

- Circulation with haemorrhage control.

- Disability: neurological status.

- Exposure/environmental control - undress the patient but prevent hypothermia.

\section{Airway with cervical spine immobilization}

If a patient is not able to maintain their own airway, for whatever reason, irreversible cerebral damage can occur in as little as 4 minutes. It is therefore essential that a safe and secure airway is established as the first step. In maxillofacial trauma or other conditions associated with immediate difficulty in gaining an airway, an emergency cricothyroidotomy can be carried out as a temporary measure. In $10-15 \%$ of poly-traumatized patients there will have been an associated spinal injury, of which $55 \%$ occur in the cervical spine.(7) A proportion will be unstable and injudicious manipulation of the spine, as may be done to secure an airway, runs the risk of spinal cord injury. Therefore an equal priority must be given to the in-line traction applied by hand on either side of the head to maintain stabilization of the cervical spine while trying to secure an airway.
If there is no evidence of airway compromise, or once an airway breathing is spontaneous, the usual procedure moves attention to next issue. The lungs are vital for maintaining oxygenation of the body tissues as well as removing waste carbon dioxide and helping to maintain acidbase balance. There are six causes of life-threatening respiratory compromise: upper airway obstruction, tension pneumothorax, open pneumothorax, flail chest, massive haemothorax and cardiac tamponade.(7)

Initial treatment consists of removing the mechanical problem (insertion of an intercostal drain for haemo/ pneumothorax; application of an occlusive dressing for sucking chest wound), providing high-flow oxygen and providing mechanical ventilator support if necessary. In patients with a suspected spinal injury, it is always important to remember the possibility of spinal shock. This occurs in patients who have a cord injury above the level of the thoracic sympathetic outflow. The haemodynamic result is hypotension and bradycardia. It is the bradycardia that tends to differentiate spinal shock from hypovolaemic shock, although patients on в-blockers or with cardiac arrhythmias may also have bradycardia. Treatment of spinal shock involves the judicious use of intravenous fluids combined with vasopressors to increase the resting vascular tone.

ATLS teaches that "trauma occurring above the clavicle should raise a high index of suspicion for a potential cervical spine injury' and strict application of this principle means all patients with maxillofacial or craniofacial trauma must be included in this group.(6)

Accordingly, maxillofacial trauma patients must be initially managed with a :

- cervical spine collar until clinical and radiological clearance is confirmed,

- comprehensive neurological examination including cranial nerves

- specific assessment for cerebrospinal fluid rhinorrhea.(4)

\section{Circulation}

Maintaining adequate tissue perfusion and hence oxygenation requires an adequate circulating blood volume, adequate vascular tone and a normally functioning heart. In the vast majority of trauma victims, the main reason for circulatory compromise is acute blood loss leading to haemorrhagic shock. However, some other causes of shock do occur and should also be considered. These include:

- cardiogenic shock

- tension pneumothorax

- neurogenic shock

- septic shock.

Haemorrhagic shock: while some bleeding may be obvious, frequently the exact source may not be readily apparent. The common sites for major occult loss are:

- the chest, in cases of haemothorax 
- the abdomen, from ruptured viscera

- the pelvis, from an unstable pelvic fracture

- from multiple closed long bone fractures.

The key early signs of haemorrhagic shock are tachycardia and cutaneous vasoconstriction. In healthy young adults, hypotension occurs later and signifies a blood loss in excess of $1500-2000 \mathrm{ml}$. The initial fluid bolus is 1-2 litres for an adult and $20 \mathrm{ml} / \mathrm{kg}$ for a child. This is followed by blood transfusion. Fully cross-matched blood tests is preferable, but in an urgent situation type-specific ( $\mathrm{ABO}$ and $\mathrm{Rh}$ matched blood) can be used. In lifethreatening hypotension, $\mathrm{O}$ negative packed cells can be used. The return of blood pressure and pulse to normal are encouraging signs. Restoration of urinary output to $0.5 \mathrm{ml} /$ $\mathrm{kg} / \mathrm{hr}$ suggests that adequate renal perfusion has been restored.(7)

\section{Disability (neurological status)}

The possibility of brain injury is assessed next. A decrease in a patient's level of consciousness may be due to a primary brain injury. This is assessed very quickly by examining the pupil size and reactivity and by assessing whether the patient is alert, responds to verbal stimuli, responds only to pain or is unresponsive. Unequal pupillary responses may be indicative of local trauma to the eye (traumatic mydriasis) or, more worryingly, an expanding intracranial haematoma. The Glasgow Coma Scale(GCS) is a useful clinical method of monitoring the status of patients following head injury. This gives a score out of 15 based on the patient's best motor, verbal and eye responses. A score of 15 would indicate a fully alert, cooperative and comprehensible patient, and a score of 8 or less usually indicates the presence of serious cranial trauma.

A decreased level of consciousness, particularly in the absence of any signs of external head injury, may represent inadequate cerebral oxygenation and perfusion, prompting a swift re-assessment of A, B and C. (5)

\section{Exposure/environment}

To facilitate a thorough whole body examination, all clothing should be removed from a patient. Frequently this requires clothing to be cut off. This allows an adequate assessment of the spinal column, the posterior aspects of the limbs and the perineum.

Once the appropriate examinations have been performed, it is important that the patient is covered with blankets to prevent hypothermia. Additional preventative measures include the warming of intravenous fluids and the maintenance of a warm environment in the resuscitation room.(7) It is vitally important that progress through the assessment and resuscitation sequence does not occur until problems of higher priority are appropriately dealt with. There is no point in trying to put on a pelvic external fixator if the patient cannot maintain his own airway. Equally, the condition of a traumatized patient is constantly changing and frequent reassessment is required to ensure there has been no deterioration.

Maxillofacial surgeons should be an integral part of the trauma team for those patients where facial injuries are evident. This involvement is particularly relevant during the management of:

1. the airway,

2. hypovolaemia including facial bleeding

3. craniofacial injuries

4. in the assessment of the eyes.(5)

If the patient's blood alcohol levels are high, they are unlikely to fall in the next $12-24 \mathrm{~h}$ and the following 'hangover' will almost certainly be associated with vomiting. If the patient's consciousness level is of concern, intubation is necessary; also, in these circumstances a CT scan of the brain is indicated.(8)

\section{SUMMARY}

- The management of polytraumatized patients remains a significant challenge, requiring a multidisciplinary approach.

- Initial assessment and treatment should follow a structured approach (ATLS), which prioritizes lifethreatening injuries.

- Due to the high frequency of maxillofacial trauma, the maxillofacial surgeon is an integral part of the trauma team. During and immediately after the 'golden hour', maxillofacial surgeons may need to provide an advisory service or to intervene in some way.

- Definitive repair procedures will depend on a number of factors related to the patient's general status, overall prognosis and other planned procedures

- Specific injury patterns are now well known to be associated with high- velocity mechanisms of injury

- Maxillofacial surgeons managing multiply injured patients should always maintain a high degree of suspicion for associated injuries, the negligence of which can have a drastic effect on outcome.

From a maxillofacial perspective, although it is the gold standard in trauma care, ATLS does have its limitations, and strict adherence to its principles in patients with facial injuries brings its own set of problems. With a greater understanding of trauma mechanisms, a multidisciplinary approach and advances in technology, many of our concerns can now be anticipated and addressed in a timely manner. 


\section{REFERENCES:}

1. American College of Surgeons Committee on Trauma (2004) Advanced trauma life support program for doctors, 7th edn. American College of Surgeons, Chicago.

2. American College of Surgeons Committee on Trauma Advanced trauma life support program for doctors, 6th edn. American College of Surgeons, Chicago.

3. Carmont MR. The Advanced Trauma Life Support part1 course: a history of its development and review of related literature. Postgrad Med J. 2005 Feb;81(952):87-91. [PubMed] [CrossRef]
4. Grover RS, Antonyshyn OM. (iv) Care of maxillofacial injuries in multiple trauma. Orthopaedics and Trauma. 2003 Aug;17(4)274-286

5. Perry M. Advanced Trauma Life Support (ATLS) and facial trauma: can one size fit all? Part 1: dilemmas in the management of the multiply injured patient with coexisting facial injuries. Int J Oral Maxillofac Surg. 2008 Mar; 37(3):209-14. [PubMed] [CrossRef]

6. Perry M, Morris C. Advanced trauma life support (ATLS) and facial trauma: can one size fit all? Part 2: ATLS, maxillofacial injuries and airway management dilemmas. Int $J$
Oral Maxillofac Surg. 2008 Apr; 37(4): 309-20. [PubMed] [CrossRef]

7. Simpson P, Keating JF. The multiply injured patients, Acute trauma, The foundation years $4: 8$, Published by Elsevier Ltd. 2008; p.314-318

8. Head Injury: triage, assessment, investigation and early management of head injury in infants, children and adults. National Collaborating Centre for Acute Care. Guideline commissioned by the National Institute for Clinical Excellence; June 2003; http://www.nice.org.uk/nicemedia/pdf/ headinjury_full_version_completed.pdf
Address for correspondence:

Elitsa Georgieva Deliverska, Department of Oral and Maxillofacial Surgery, Faculty of Dental Medicine, Medical University - Sofia. 1, St. Georgi Sofiiski boul., 1431 Sofia, Bulgaria E-mail: elitsadeliverska@yahoo.com; 